\title{
CONTINUUM LIGHT FROM SUPERNOVAE
}

\author{
Robert P. Kirshner \\ Department of Astronomy \\ University of Michigan \\ Ann Arbor, Michigan 48109
}

Recent empirical and theoretical work is based on the idea that both Type I and Type II supernovae emit a thermal continuum near maximum light. Because this interpretation is so simple, it allows the flux distribution observed through spectrophotometry ${ }^{1-5}$ to be transformed in to the physical quantities of radius and temperature, which can be compared with theoretical expectations. . $^{6-8}$

Supernovae have always provided a large arena for theoretical speculation, but as indicated in FIGURE 1, it is important to be scientific, like Ol' Doc Dabble, and to check on those "figgers."

For Type II supernovae, which have been found only in spiral galaxies, and which are presumed to result from the explosion of massive stars, the continuum is well defined. Although no spectrophotometry has yet been obtained of a Type II supernova before maximum light, the available evidence favors a temperature at maximum between $9000^{\circ} \mathrm{K}$ and $20,000^{\circ} \mathrm{K}$, with rapid cooling to about $5000^{\circ} \mathrm{K}$ $6000^{\circ} \mathrm{K}$ in the 30 days after maximum light (Figure 2.) As shown in Figure 3, the apparent continuum temperature remains constant for another 100 days. At later times, the continuum is very poorly determined, although it is consistent with a temperature near $5000^{\circ} \mathrm{K}$. By assuming a distance to the supernova based on the red-shift of the galaxy in which it occurred, the apparent flux density and temperature can be used to calculate the photospheric radius as a function of time, as shown in Figure 4. As seen in that figure, the photosphere expands for more than 50 days after maximum light, up to a radius of about $1.6 \times 10^{15} \mathrm{~cm}$. The expansion is remarkably linear, with a mean expansion rate of $4600 \mathrm{kms}^{-1}$ over the first 40 days. Of course, as the ejected material expands it becomes increasingly transparent. The fact that the velocity at which the photosphere expands does not change requires that a substantial amount of the ejected mass is at a velocity of about $4600 \mathrm{kms}^{-1}$, presumably in a shell. Once the shell starts to turn transparent, the photosphere recedes rapidly in Lagrangian coordinates. Although it would be extremely interesting to observe the structure of the shell through this phase, no suitable data exist. It is no coincidence that the broad-band magnitudes of Type II supernovae are often observed to take a drastic plunge after about 50-70 days. This is no doubt the same phenomenon of the envelope turning transparent, observed in a more primitive way.

Even though the exterior vanishes from sight, as Figure 3 demonstrates, a continuum is still formed, and the central object (if there is any) continues to be hidden from view. The photospheric radius is about $3 \times 10^{14} \mathrm{~cm}$, and the average expansion velocity for the gas at the photosphere must be about $275 \mathrm{kms}^{-1}$.

Measurement of the apparent displacement of the Balmer lines as a function of time yields the variation of the velocity at the place where those lines are formed. As Figure 5 shows, at early times that velocity is considerably higher than the 


\section{Be Scientific with OL' DOC DABBLE.}

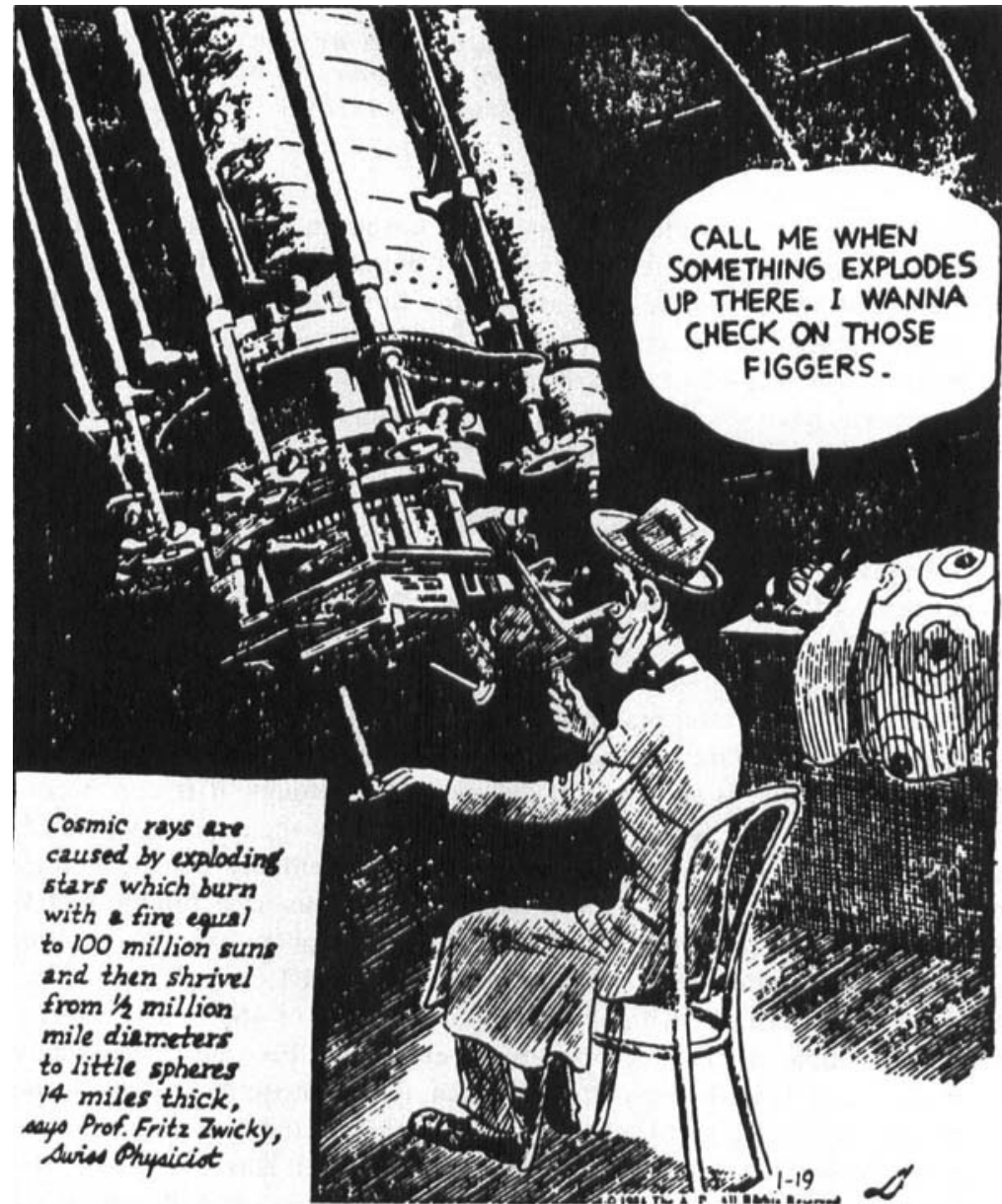

Figure 1. This cartoon appeared in the Los Angeles Times for January 19, 1934. In the box at the left is one of the most concise predictions of supernova properties ever published. The prediction of neutron stars in supernova explosions ("Iittle spheres 14 miles thick") is especially interesting. Ol'Doc Dabble presents a model of empiricism, as he anticipates the mode of operation of modern observational astronomers, both by his readiness to observe, and his willingness to let someone else search for the supernovae!

average photospheric expansion velocity. This is the natural effect of higher opacity in the lines than in the continuum in an atmosphere where the highest velocity material is at the outside. This opacity effect is also responsible for the $\mathrm{H} \alpha$ velocity exceeding $\mathrm{H} \beta$, which is larger than the velocity at $\mathrm{H} \gamma$. At a time of about 50 days, just when the shell that forms the photosphere turns transparent, the 
velocity measured in the lines is just about the same as the mean photospheric expansion of $4600 \mathrm{kms}^{-1}$ ! At that time, the lines are presumably being formed in the dense shell region. That interpretation is reinforced by the subsequent slow change in line velocity.

As utilized by Kirshner and $\mathrm{K}$ wan, ${ }^{3}$ the distance to the supernova need not be assumed but can be derived by matching the relative expansion of the photosphere to the absolute expansion of the envelope as determined from the absorption lines. This method of finding extragalactic distances has the unique property that it is independent of all distances determined in our own galaxy, such as those of the Hyades, the RR Lyraes, and the Cepheids.

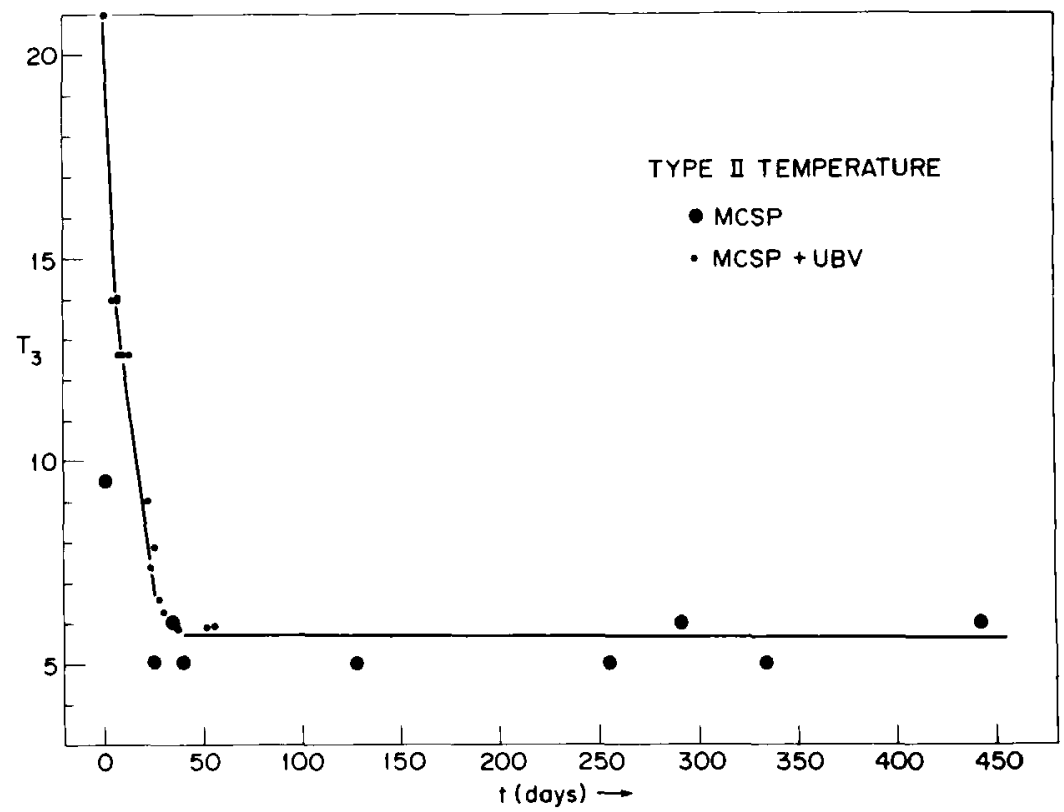

Figure 2. Continuum temperature, as derived either from multichannel spectrometer (MCSP) scans or from a combination of MCSP scans and broad-band UBV photometry.

The physical quantities presented in Figures 2, 4, and 5 can be compared with theoretical calculations carried out by $\mathrm{Chevalier}^{6}$ and by Falk and Arnett. ${ }^{7}$ These calculations are based on our current ideas of the nature of stars of several solar masses near the end of stellar evolution. In both cases, the details of the supernova detonation are ignored. All that is assumed is that $10^{50}-10^{51}$ ergs is deposited at the inside of the envelope. Then a hydrodynamic code is used to follow the disruption of the star. The remarkable fact is that the observed radius, temperature, and velocity can be reproduced without excessive adjustment of the parameters. The principal difference between the Chevalier models and those of Falk and Arnett is that in the former the shock emerges from the surface days before maximum light, 
whereas in the latter the maximum is nearly coincident with the appearance of the shock. The observational test to distinguish the two cases requires spectrophotometry of a Type II supernova right through the peak of luminosity. This will require unusually prompt action on the part of observers. These models are different in concept from the pulsar-powered model of Bodenheimer and Ostriker. ${ }^{10}$

Figure 4 shows one of Chevalier's models, which employs a fairly realistic model of the stellar density distribution. Although the radius reached in the model is somewhat larger than observed, the general features of a linear increase to a few $10^{15} \mathrm{~cm}$, rapid drop at 50 days, and slowly changing radius of a few $\times 10^{14} \mathrm{~cm}$ at late times are admirably reproduced.

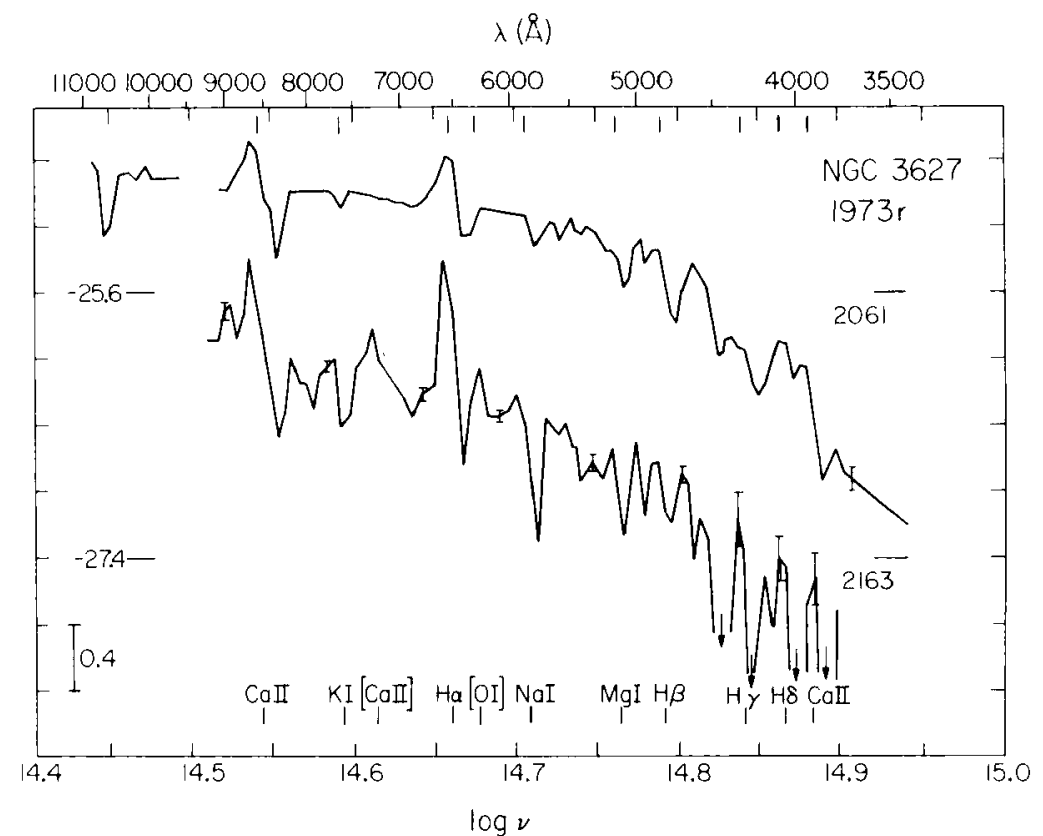

Figure 3. MCSP scans for the type II supernova $1973 \mathrm{r}$ in NGC 3627. The two sets of data are taken at 26 and 128 days past maximum light. Note the continuing presence of a continuum with a temperature near $5000^{\circ} \mathrm{K}$.

Two features of the presupernova stars are responsible for the success of these models. One is that the stars are very extended, so that the adiabatic losses in expanding to $10^{15} \mathrm{~cm}$ are not devastating. A second is that the stars have a long region in their envelopes in which the density is nearly constant. This permits efficient transport of the energy of the explosion out through the star.

Type I supernovae show striking similarities to Type II in their temperatures, radii, and velocities. As exhibited in FIGURE 6, the photospheric temperature of 


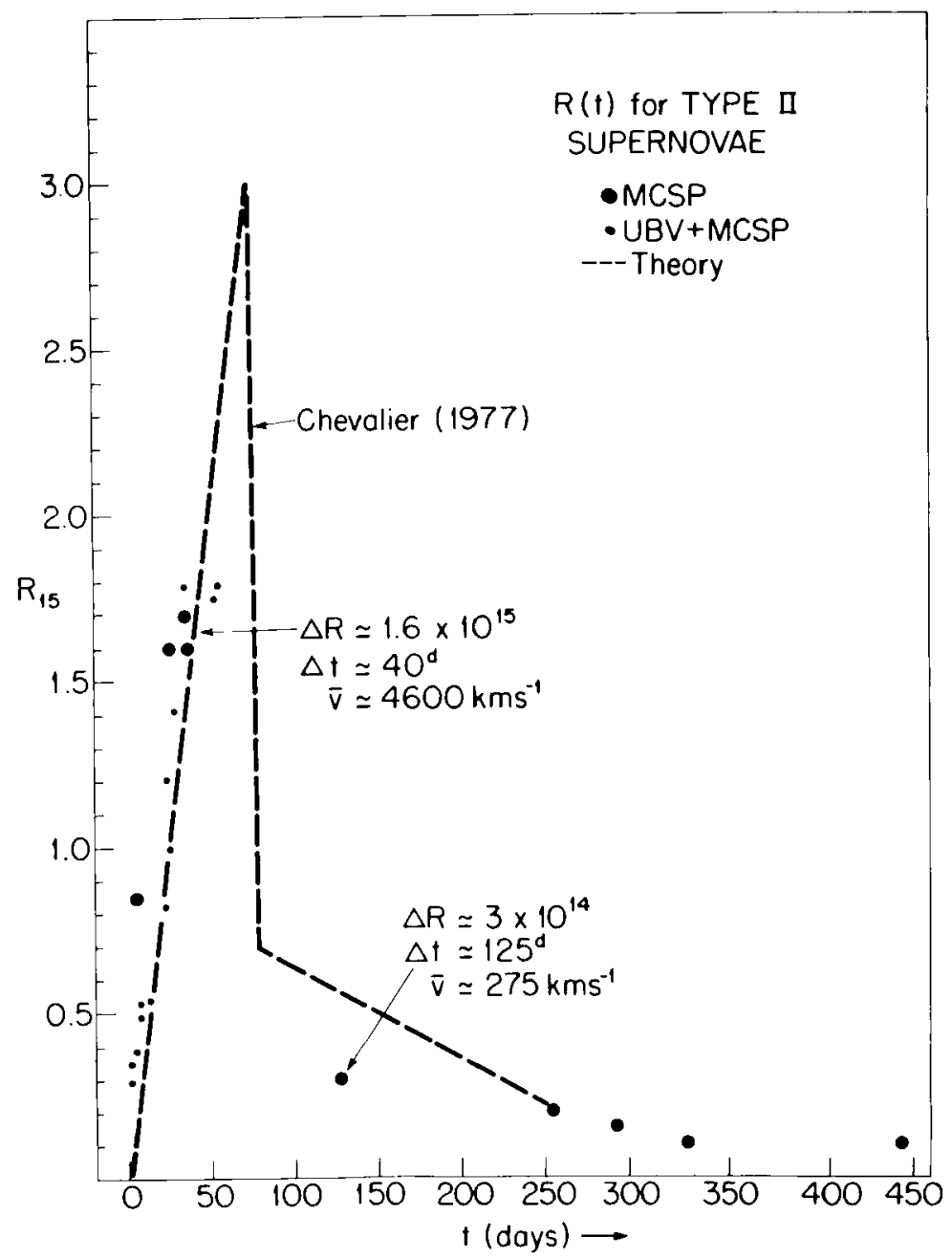

Figure 4. Radius of the photosphere of Type II supernovae. Data from three supernovae are combined here to provide a picture of the photosphere's motion. The early expansion, at roughly $4600 \mathrm{kms}^{-1}$, persists until a radius of about $1.6 \times 10^{15} \mathrm{~cm}$ is reached. Then the envelope, which is presumably in the form of a shell, rapidly turns transparent and appears to shrink. At an age of roughly 100 days, the continuum is still present (as shown in Figure 3), but much reduced in radius. The average velocity of the material forming the photosphere at that time is about $275 \mathrm{kms}^{-1}$. In a realistic model for Type II envelopes by Chevalier (shown with the broken line), these features are reproduced with very good qualitative and fairly good quantitative agreement. 


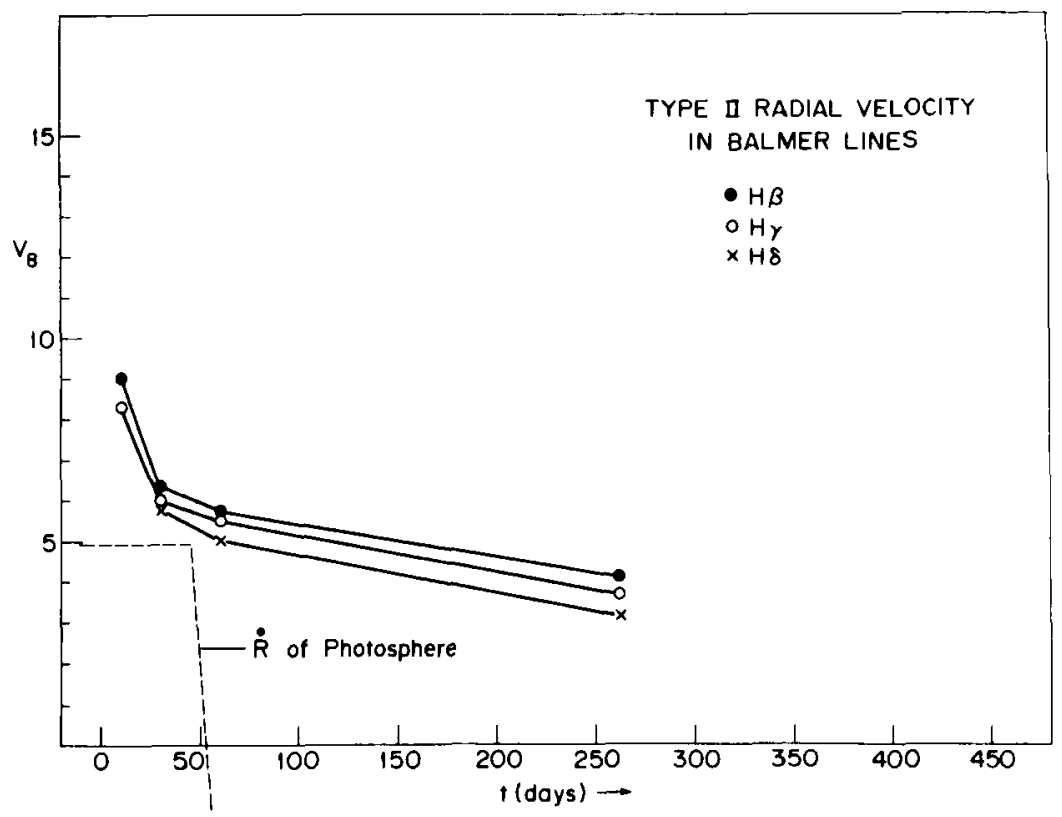

Figure 5. Velocity measured from Balmer lines in spectra of Type II supernovae. These lines are formed well above the photosphere at early times, but after the photosphere starts to turn transparent at age 40 days, the velocity is comparable to the mean photospheric velocity of $4600 \mathrm{k} \mathrm{ms}^{-1}$. This may be the result of a large amount of mass at this velocity-in the form of a dense shell.

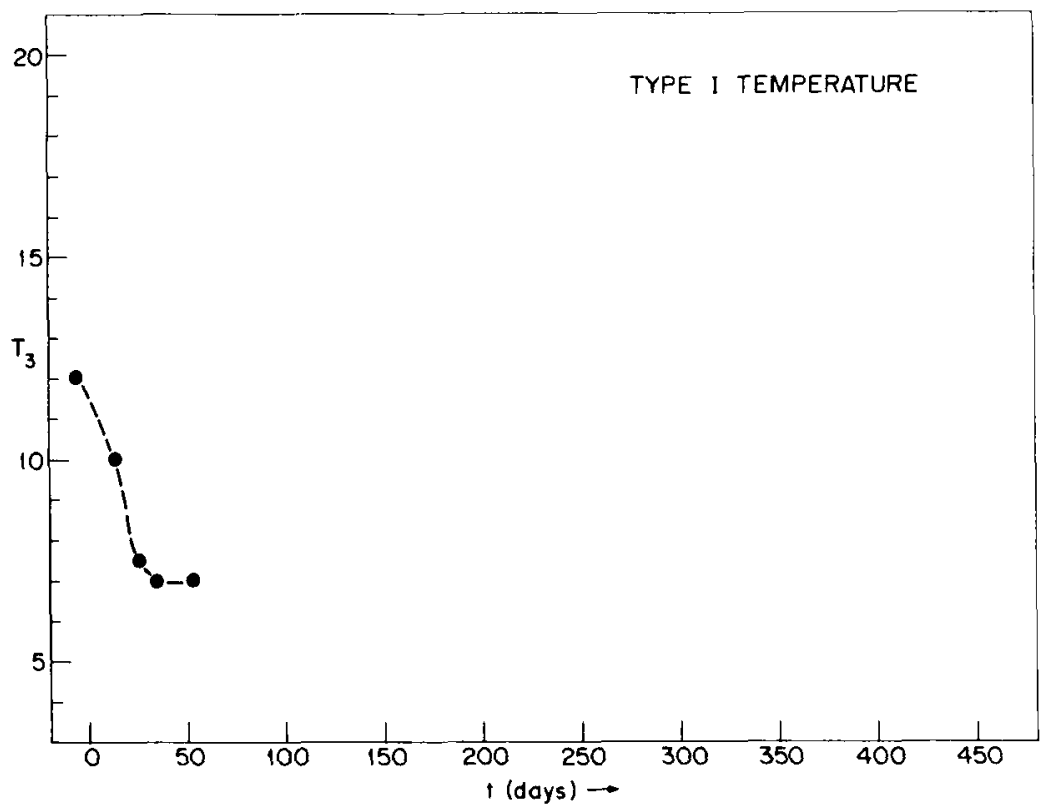

Figure 6. Temperature of the photosphere for Type I supernovae. The point at 3 days before maximum shows a temperature of only $12,000^{\circ} \mathrm{K}$. 
Type I supernovae goes from about $12,000^{\circ} \mathrm{K}$ at maximum light down to about $7,000^{\circ} \mathrm{K}$ in a span of 50 days. After that point, the continuum is very poorly determined by observations. The famous exponential decline of Type I supernovae is a phenomenon of their emission lines and not of their continua.

The radius at maximum light for the well-observed Type I in NGC 2207 was about $1.0 \times 10^{15} \mathrm{~cm}$, as compared with about $3.0 \times 10^{14} \mathrm{~cm}$ for Type $11 .{ }^{5}$ The subsequent expansion of the photosphere to $1.6 \times 10^{15} \mathrm{~cm}$ is about the same extent as seen for Type II. Figure 7 shows the behavior of the photosphere for Type I supernovae. For Type $\mathbf{I}$, the velocity measured in the Call $\mathbf{H}$ and $\mathrm{K}$ lines is about

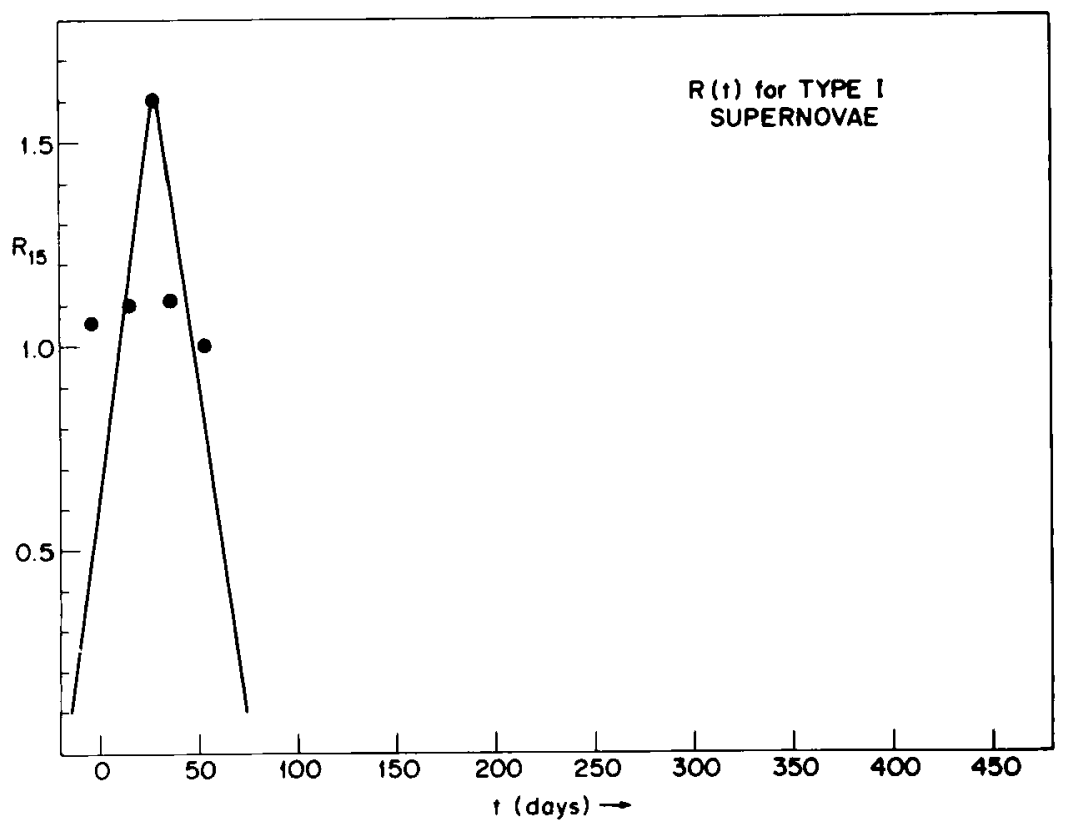

Figure 7. Photospheric radius of Type I supernovac. The present observations do not yet show the history of the expansion in a very detailed way, but the maximum radius reached seems very similar to that for Type II.

$11,000-14,000 \mathrm{kms}^{-1}$ near maximum light, somewhat larger than the value of $9,000 \mathrm{kms}^{-1}$ observed for Type II. Although the data are not very detailed at late times, they indicate no change in the absorption line velocity after 50 days, as shown in Figure 8. This is reminiscent of the behavior attributed to a shell for Type Il line velocities.

The similarity between the properties of Type I and Type II continua raises the question of whether they arise from the same causes. Lasher $e t$ al. have assumed that the extended stars with constant-density envelopes are the presupernova stars 
for Type I just as for Type II. ${ }^{8}$ Their hydrodynamic calculations show that the observable features of Type I supernovae can be matched fairly well in this way. The presence of Type I supernovae in elliptical galaxies is quite puzzling under this model, since very extended stars, which are thought to be short-lived and massive stars, are required. Nevertheless, the similarity of Type I and Type II continuum features is very strong and might reasonably be assigned the same cause. If that is true, then the possibility that Type I supernovae come from very extended stars has to be dealt with properly.

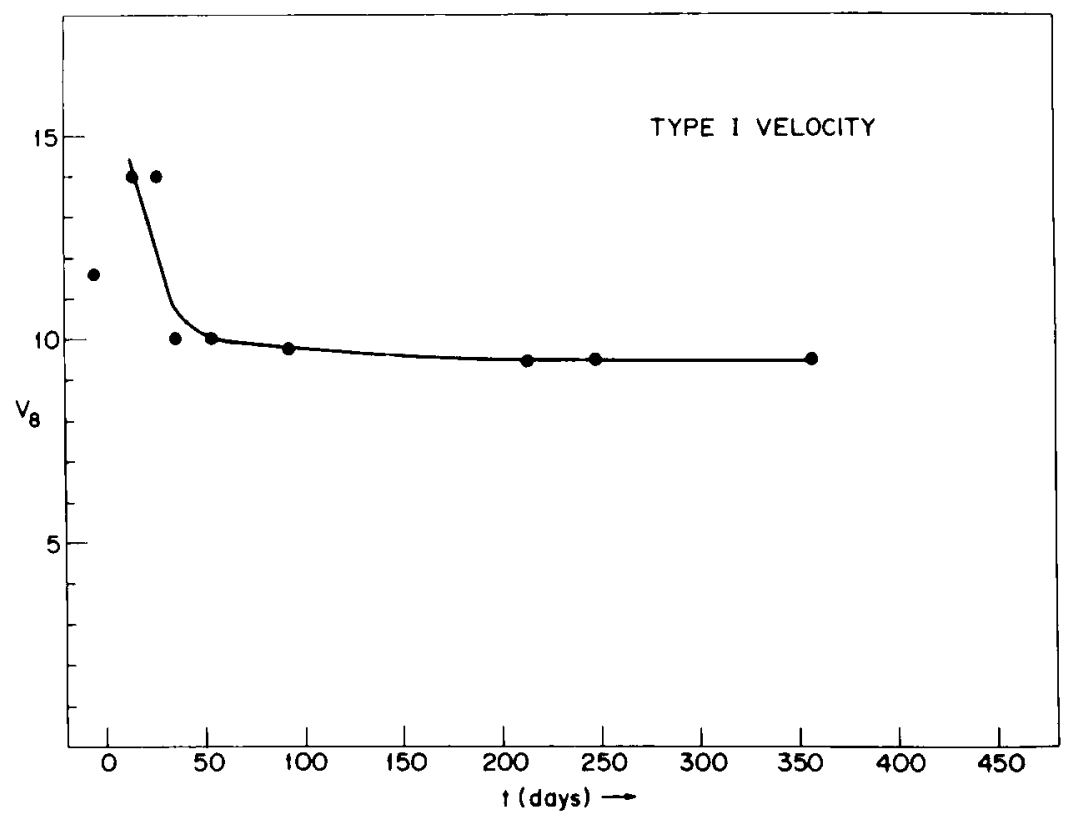

Figure 8. Radial velocity measured from Call lines in Type I spectra. The behavior that was attributed to a dense shell in Type II is also seen here.

Just as for the Type II, the prospects of determining extragalactic distances from Type I supernovae presents an intriguing possibility. "If we can gather adequate data in the first month of evolution for a dozen Type I supernovae, we may be able to make some contribution to the distance scale at distances of $10-100$ Mpc. However, there is at present no observatory with the instrumentation to do this, that is committed to obtaining supernova spectra of every accessible discovery. We hope that the 52-inch telescope at the McGraw-Hill Observatory on Kitt Peak can be used with its new photon-counting spectrograph to obtain the required data. 


\section{REFERENCES}

1. Kirshner, R. P., S. P. Willner, E. E. Becklin, G. Neugebauer \& J. B. Oke. 1973. Astrophys. J, Lett, 180: L97.

2. Kirshner, R. P.. J. B. Oke, M. V. Penston \& L. Searle. 1973. Astrophys. J. 185: 303.

3. Kirshner, R. P. \& J. KWAN. 1974. Astrophys. J. 193: 27.

4. Kirshner, R. P. \& J. Kwan. 1975. Astrophys. J. 197: 415.

5. Kirshner, R. P., H. C. Arp \& J. R. Duni.aP. 1976. Astrophys. J. 207: 44.

6. Chevalier, R. A. 1977. Astrophys. J. In press.

7. Arnett, W. D. \& S. W. Falk. 1976. Orange Aid Preprint. Calif. Inst. of Tech. Pasadena, Calif.

8. LASHER, G. 1975. Astrophys. J. 201: 194.

9. Branch, D. \& B. Patchett. 1973. Mon. Not. R. Astron. Soc. 161: 71.

10. Bodenheimer, P. \& J. P. Ostriker. 1974. Astrophys. J. 191: 465. 